\title{
Thromboresistance of functionalized poly(methylmethacrylate): the effect of surface polarity
}

\author{
GOVINDA KAPUSETTI, MONIKA, AMIT KUMAR RAY and NIRA MISRA* \\ School of Biomedical Engineering, Indian Institute of Technology, Banaras Hindu University, Varanasi 221 005, India
}

MS received 5 March 2014; revised 25 June 2014

\begin{abstract}
An implant material when comes in contact with blood fluids (e.g., blood and lymph), adsorb proteins spontaneously on its surface. Notably, blood coagulation is influenced by many factors, including mainly chemical structure and polarity (charge) of the material. The present study describes the methodology to improve the blood compatibility of poly(methylmethacrylate) (PMMA) by incorporating ionic groups with varying polarities. PMMA has been functionalized with different groups containing positive, negative and neutral polarity by the free radical polymerization technique and such modification were further confirmed through Fourier transform infrared (FTIR) spectroscopy. The level of thrombogenicity was found three times lower with negatively charged PMMA in comparison to those of positively charged and neutral PMMA. Platelet adhesion was noted almost negligible in all samples after $10 \mathrm{~s}$ of blood exposure. High adsorption of fibrinogen from the blood was noticed in the test sample containing a group with positive polarity (thiouronium chloride) while there was no platelet adhesion observed even after $120 \mathrm{~s}$ of blood exposure in the test samples containing negatively charged (sulphate) and neutral (hydroxyl group) functional groups.
\end{abstract}

Keywords. Thrombogenicity; functional group; poly(methylmethacrylate); surface charge; sulphonate.

\section{Introduction}

Blood coagulation is a natural life saving procedure involves platelet as an important mediator to stop haemorrhage. ${ }^{1}$ When a foreign material is exposed to blood or tissue fluids, it immediately adsorbs the biomolecules and cells, primarily proteins. $^{2}$ Thus, the interaction of a polymer with the blood results into the deposition of proteins, platelets and other elements onto the polymer surface. Proteins deposit onto the polymer surface immediately upon contact with the blood, while the platelets start to adhere at least 1 min after contact with the blood (i.e., when the thickness of protein layer is around $200 \AA$ ). The conversion of fibrinogen to fibrin, and the activation and aggregation of platelets lead to thrombus formation and growth on the artificial surface. ${ }^{3}$

The blood compatibility of the biomedical surfaces is greatly dependent upon their physical and chemical characteristics. Attempts to improve antithrombogenicity of polymer surface have been made by many investigators covering various aspects, including negative surface charge, interfacial free energy, charge degree, balance of hydrophilicity/ hydrophobicity and surface morphology. ${ }^{4,5}$ Thromboresistant materials are generally characterized by their surface smoothness, improved semiconductivity, inertness and surface properties (i.e., magnitude of the negative charge) of the modified material. Approaches for such developments include chemical modification by the surface grafting with

*Author for correspondence (nira1953@yahoo.co.in) hydrogels, ${ }^{6}$ deposition of an ultrathin layer of a polymer by the plasma treatment ${ }^{7}$ and incorporation of an ionic group to the polymer surface. ${ }^{3}$ Hydrophilic polymers are hypothesized to be more antithrombogenic due to a minimal interaction with the blood. ${ }^{8}$

It has been well documented that electrical charge on the polymer surface plays an important role in blood compatibility. Notably, the electrical repulsion between blood components and the negatively charged blood vessel's wall promotes the thromboresistance of the material. ${ }^{9}$ It has been reported that positively charged surfaces are generally thrombogenic, while uniformly negative charged surfaces, i.e., similar to characteristics of the blood vessel's wall tend to be antithrombogenic. ${ }^{10}$

Numerous functional groups have been utilized in order to render negative charge polarity to the polymers. Amongst them, sulphonate group has attracted a great attention owing to its high thromboresistance. ${ }^{11,12}$ It is well known that the unique anticoagulant property of heparin is mainly attributed to its associated aminosulphate and sulphate groups. Similarly, polymers containing sulphonate groups such as sulphonated polystyrene, ${ }^{13}$ poly(hydroxyethylmethacrylate) HEMA-sulphoxyl (methacrylates) ${ }^{14}$ and sulphonated polyurethanes ${ }^{15,16}$ have shown enhanced blood compatibility and high thromboresistance that might be attributed to the decreased adhesion of platelets.

Acidic and basic functional groups of polymers respond to basic (methylene blue) and acidic (di-sulphide blue) dyes, respectively. ${ }^{17,18}$ The presence of $0.1 \mathrm{M}$ of such functional 
groups renders the whole polymer either negatively or positively charged through the inductive effect. Thus, the polymer are characterized by their surface charge, i.e., positive, negative and neutral depending on the integrated functional moieties. ${ }^{18}$ Polymeric surfaces are generally divided into two categories when exposed to the blood: one that adsorbs albumin and where the resulting polymeric surface repels platelets, and another group of polymer that adsorbs $\gamma$-globulin and fibrinogen and promotes the formation of thrombus by facilitating the adhesion of platelets. ${ }^{19}$ There are numerous studies reported on the interaction of blood with the polymer surface. ${ }^{20}$ The present study describes preparation of three types of polymeric surface modified with positive, negative and neutral functionalities using distinct types of initiator systems, including (i) ferric ammonium sulphate, thiourea system, (ii) persulphate, metabisulphite system and (iii) ferrous ammonium sulphate, hydrogen peroxide, respectively, for methylmethacrylate polymerization.

\section{Experimental}

\subsection{Materials}

Ferric ammonium sulphate, ferrous ammonium sulphate, thiourea, potassium persulphate, potassium metabisulphate, hydrogen peroxide, gluteraldehyde, sodium chloride all were GR grade and procured from Merck, India Ltd.

\subsection{Synthesis}

Standard aqueous polymerization method was followed for preparation and purification of poly(methylmethacrylate) (PMMA) with thiouranium, ${ }^{21}$ sulphonate $^{22}$ and hydroxyl groups. Films of these three polymers were prepared on round brass plate $(1 \mathrm{~cm}$ diameter) by the solvent evaporation technique from benzene.

\subsection{Characterization}

2.3a Fourier transformation of infrared spectroscopy (FTIR): FTIR technique was applied to detect the functional groups and to understand the nature of interaction between PMMA and the functional groups. FTIR was performed in transmittance mode at room temperature from 400 to $4000 \mathrm{~cm}^{-1}$ using Nicolet 670 FTIR with a resolution of $4 \mathrm{~cm}^{-1}$. The bubble-free thin films were prepared by dissolving in tetrahydrofuran (THF) solvent with a special care.

2.3b Thrombosis assay: Hyparinized films were hydrated to equilibrium with $0.5 \%$ saline water and kept at $37^{\circ} \mathrm{C}$ for a while in Petri dishes followed by the addition of $0.2 \mathrm{ml}$ ACD human blood to each film. Blood clotting was initiated by the mixing of $0.02 \mathrm{ml} \mathrm{of} \mathrm{m} / 10 \mathrm{KCl}$ solution. Clotting was stopped by adding $5 \mathrm{ml}$ of distilled water after $15 \mathrm{~min}$. Clot was set in $5 \mathrm{ml}$ of $36 \%$ formaldehyde solution for $5 \mathrm{~min}$. Predetermined clot was washed with distilled water and blotted between tissue paper and weighed.

2.3c Platelet adhesion: Three films for each functionalized polymer was subjected to blood, obtained through puncturing finger tip of healthy man for $0-120 \mathrm{~s}$. Washed with $0.9 \%$ saline water and platelets were fixed by washing with $0.2 \%$ gluteraldehyde solution films were thus coated with gold coater and were subject to scanning electron microscopy (SEM) in a LEO 435VP instrument operated at $10 \mathrm{kV}$.

2.3d Statistics: The results were expressed as mean values ( \pm SEM). The analysis of variance followed by a post hoc Dunnett's multiple comparison tests was performed for thrombosis assay. In all cases, $p<0.05$ was considered to be significant (GRAPH PAD PRISM 5.1).

\section{Results and discussion}

Figure 1 shows the FTIR spectrograms of PMMA and its surface modified systems. Two new transmission peaks corresponding to the symmetric stretching band of aromatic $-\mathrm{SO}_{3} \mathrm{H}$ group and $\mathrm{C}-\mathrm{S}$ stretching vibration were appeared at 1061 and $655 \mathrm{~cm}^{-1}$, respectively, in sulphonated PMMA in figure $1 \mathrm{a}^{23}$ Figure $1 \mathrm{~b}$ shows the hydroxyl group substituted PMMA FTIR spectrogram; a new broad peak was observed at and it subjected to - $\mathrm{OH}$ stretching, which reveals the presence of hydroxyl group in modified PMMA. Two new peaks appeared at 3330 and $3240 \mathrm{~cm}^{-1}$ in thiouronium chloride functionalized PMMA and these peaks are

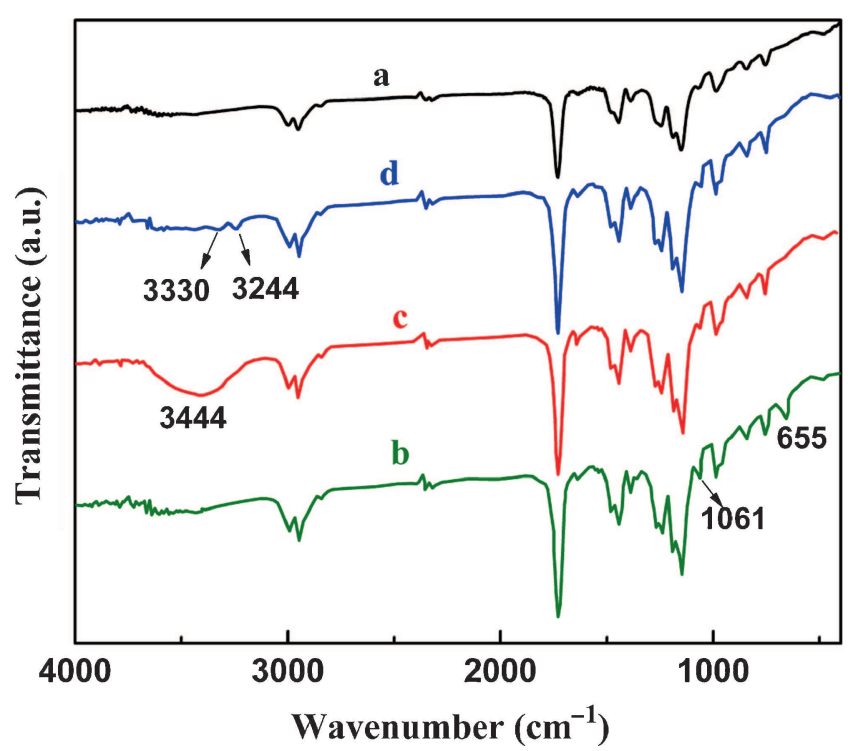

Figure 1. The FTIR spectrograms (a) PMMA, (b) sulphonated PMMA, (c) hydroxyl PMMA and (d) thiouronium chloride functionalized PMMA. 
responsible for the $\mathrm{N}-\mathrm{H}$ asymmetric and symmetric stretching, respectively. ${ }^{24}$ Thus, the results suggest that functional groups have been successfully bonded with PMMA chains.

\subsection{Thrombosis assay}

Heparinized PMMA and its functionalized films were subjected to human blood for measuring the thrombogenicity for a time interval of $15 \mathrm{~min}$. The weight of the clotted blood when exposed to negatively polarized sulphonated PMMA, neutral polarized hydroxyl PMMA and positively polarized thiouronium chloride PMMA thin films was estimated about $0.52 \mathrm{mg}(P<0.001), 0.89 \mathrm{mg}$ and $1.67 \mathrm{mg}$, respectively, in comparison to the pure PMMA film (1.37 mg; figure 2). It is known that the surface polarity plays an important role at the molecular level in surface-induced thrombosis. The inhibition of blood clotting by the polymer films was mainly dependent on the degree of adverse interaction of blood components due to protein denaturation. Therefore, the improvement in blood compatibility of the sulphonated PMMA was noticed most likely due to its negative charge polarity that closely resembles to the physiological conditions of the blood vessels.

\subsection{Platelet adhesion}

Numerous studies have been conducted on the exposure of the blood to the polymeric surface coated with a single protein, albumin, fibrinogen and $\gamma$-globulin. The absorption of water molecule and small inorganic ions precedes the adsorption of plasma protein. ${ }^{25,26}$ Such phenomenon is known to occur rapidly and also significantly influences the subsequent interaction of polymeric surface with the assembled blood components, especially the platelets. The degree of platelets activation resulting from exposure of the blood to a given polymeric surface was evaluated by comparing the morphological changes in the platelets adhered to the unexposed polymer sample using SEM.

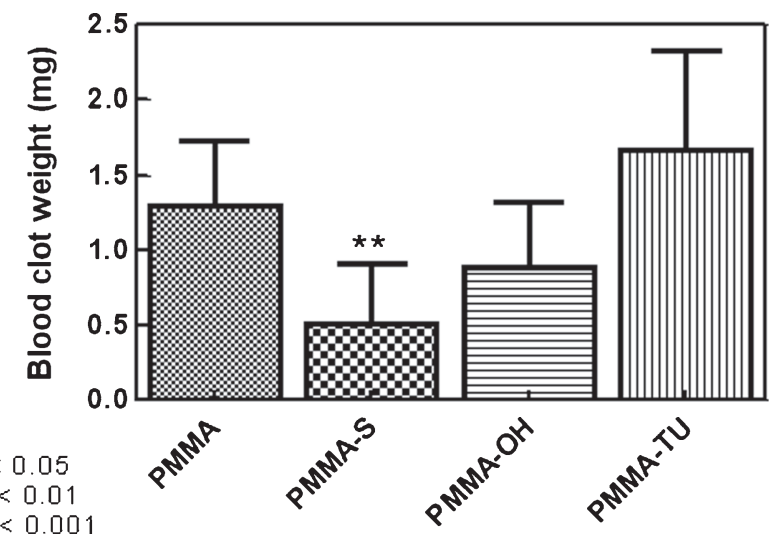

Figure 2. Bar diagram of thrombosis assay of PMMA and its functionalized counterparts with variance $\left({ }^{*} P<0.05\right)$.
Figure 3a-d shows the SEM morphology of PMMA, platelets adhered to sulphonate, hydroxyl and thiouronium chloride functionalized PMMA films, respectively, upon $120 \mathrm{~s}$ of blood exposure and table 1 shows the state of platelet adhesion as a function of time (0, 10 and $120 \mathrm{~s})$.

Figure $3 \mathrm{~b}$ and $\mathrm{c}$ reveals that the sulphate group that contains net negative charge exhibits little response towards platelet adhesion. Similar results have been observed in case of neutral charged hydroxyl group. Thiouronium chloride functionalized PMMA films show high platelet adhesion due to its net positive charge on their surface that is responsible for adsorption of fibrinogen from blood, as shown in figure $3 \mathrm{~d}$.

The force involved in adsorption is either physical (Van der Waals) or chemical (chemisorptions). The former involves dispersion and electrostatic forces, resulting from dipoles. Dispersion forces are arisen from the electric moment in atoms induced by the charges in the electron density of
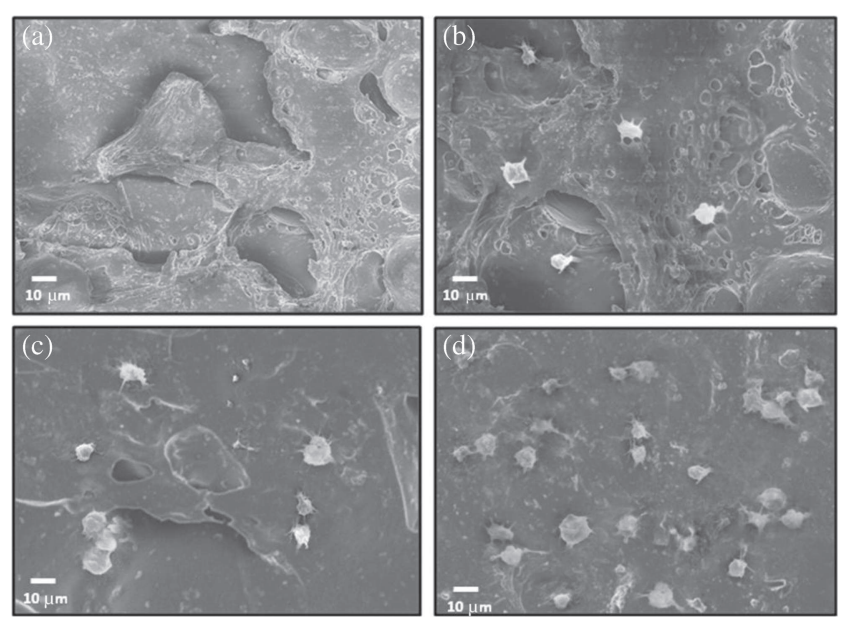

Figure 3. SEM micrographs of (a) PMMA, blood exposure for $120 \mathrm{~s}$ on functionalized PMMA specimens, (b) sulphate functionalized, (c) hydroxyl functionalized and (d) thiouronium chloride functionalized.

Table 1. Platelet adhesion of different groups with different time intervals.

\begin{tabular}{lccc}
\hline Sample no. & Group & Coagulation time (s) & Result \\
\hline 1 & Sulphonate & 0 & $-\mathrm{a}$ \\
& & 10 & $-\mathrm{a}$ \\
& & 120 & $-\mathrm{a}$ \\
& & 0 & $-\mathrm{a}$ \\
& & 10 & $-\mathrm{a}$ \\
& & 120 & $+\mathrm{a}$ \\
& Hydroxyl & 0 & $-\mathrm{a}$ \\
& & 10 & $-\mathrm{a}$ \\
& & 120 & $+++\mathrm{b}$ \\
\hline
\end{tabular}

-a: No platelet adhesion; +a: low platelet adhesion; $+\mathrm{b}$ : high platelet adhesion. 
neighbouring atoms. Hence, we infer that thiouronium chloride functionalized PMMA sample surface is favourable for adsorption process due to its electrostatic forces.

\section{Conclusions}

PMMA has been surface modified with varying polarity functional groups by the solvent evaporation technique and their structures are further confirmed through FTIR analysis. Incorporation of negative functional groups like sulphonates to the polymers even at the concentration of $0.1 \mathrm{M}$ renders it antithrombogenic, while integration of positive functionality, i.e., thiouronium groups at similar concentration induces adsorption of fibrinogen followed by the platelet adhesion. Thrombogenicity was significantly low in case of negatively charged sulphonated PMMA $(P<0.01)$. This observation was well supported by the physiological conditions of human blood vessels. However, in case of positively charged PMMA films, thrombosis was remarkably high. Thus, the present investigation reveals that the negatively charged polymer surface offers significantly low or almost negligible thrombosis effect.

\section{Acknowledgement}

Govinda Kapusetti gratefully acknowledges financial support from the Council of Scientific and Industrial Research (CSIR), Government of India, in the form of fellowship.

\section{References}

1. Rosanna A, Gabriele C, Ilaria R, Marco M and Anna M G 2012 Thromb. Res. 129235

2. Cameron J W, Richard E, Clegg D I L and Mark J P 2005 Tissue Eng. 111

3. Chen H, Yuan L, Song W, Wu Z and Li D 2008 Prog. Polym. Sci. $\mathbf{3 3} 1059$
4. Kim S W and Feijen J 1985 Biocompatibility 1229

5. Ito Y and Imanishi Y 1989 Biocompatibility 545

6. Toru M, Yoshio T, Kazuhiko I, Tomohiro K, Yorinobu T, Tomiharu M, Ung-il C, Kozo N and Hiroshi K 2004 Nat. Mater. 3829

7. Chu P K, Chen J Y, Wang L P and Huang N 2002 Mater. Sci. Eng. 36143

8. Andrade J D 1973 Med. Instrum. 7110

9. Sandip S, Kevin M S, George H and Alexander M S $2007 \mathrm{~J}$. Biomed. Mater. Res. Part B $\mathbf{8 2} 100$

10. Srinivasan S and Sawer P N 1971 In Biomedical polymers (eds) A Rembaun and M Shen (New York: Marcell Dekker) p 51

11. Mathias U 2006 Polymer 472217

12. Shen C H and Lin J C 2010 Colloid Surf. B: Interfaces 79156

13. Douzon C, Kanmangne F M, Serne H, Labarre D and Jozefowicz M 1987 Biomaterials 8190

14. Chen W Y and Feng X D 1982 J. Polym. Sci.: Polym. Chem. Ed. 20547

15. Lelah M D, Pierce J A, Lambrech L K and Cooper S L 1985 J. Colloid Interface Sci. 104420

16. Grasil J G and Cooper S L 1989 J. Biomed. Mater. Res. 23311

17. Palit S R and Mandal B M 1968 Rev. Macromol. Chem. 2225

18. Mitchell J 1992 In Applied polymer analysis and characterization (ed) J Mitchell Jr. (Munich, Vienna, New York: Hanser Publishers) vol. 2, Chapter 11B, p 58

19. Bruck S D 1975 Polymer 1625

20. Bantjes A 1978 Br. Polym. J. 10267

21. Mandal B M, Nandi U S and Palit S R 1969 J. Polym. Sci. 71407

22. Misra N, Banthia A K and Mandal B M 1979 Eur. Polym. J. 15 943

23. Guan R, Gong C, Lu D, Zhou H and Lu W 2005 J. Appl. Polym. Sci. 981244

24. Misra N, Panda H S, Kapusetti G and Jaiswal S 2011 J. Polym. Mater. 28141

25. Kazuhiko I, Kikuko F, Yasuhiko I and Nobuo N 1999 Biomaterials 201553

26. Keiji F, Hideki T, Yohichi U and Yoshito I 1993 Biomaterials 14442 